\title{
INTERACTION OF CALCIUM AND NEOMYCIN WITH ANIONIC PHOSPHOLIPID-LECITHIN LIPOSOMES
}

\author{
A DIFFERENTIAL SCANNING CALORIMETRY STUDY
}

\author{
Barbara M. Wang, ${ }^{*}$ Norman D. Weiner, ${ }^{*} \dagger$ M. G. Ganesan* and Jochen Schacht \\ ${ }^{*}$ College of Pharmacy and $\ddagger$ Kresge Hearing Research Institute, The University of Michigan, Ann Arbor, \\ MI 48109 , U.S.A.
}

(Received 6 October 1983; accepted 30 March 1984)

\begin{abstract}
The interactions of calcium and neomycin with liposomes of various anionic phospholipids plus lecithin were studied by differential scanning calorimetry. Phosphatidylinositol bisphosphate differed from other acidic phospholipids in its interactions with both calcium and neomycin. Calcium, at concentrations as low as $1 \mathrm{mM}$, induced the appearance of a second transition peak in phosphatidylinositol bisphosphate-enriched liposomes only. Neomycin acted antagonistically and precluded this phase separation. In addition, neomycin lowered the phase transition temperature of phosphatidylinositol bisphosphate-lecithin liposomes while it raised the transition temperature of all other anionic phospholipid-lecithin liposomes tested. I'his fluidizing effect of neomycin and the antagonism to calcium may induce critical alterations of properties of biological membranes. The study supports and extends our previous findings and conclusions that phosphatidylinositol bisphosphate may play a crucial role in the expression of aminoglycoside toxicity.
\end{abstract}

The ototoxicity (and possibly also the nephrotoxicity) of aminoglycosides may be based on specific membrane actions of these drugs. A number of studies on cochlear and renal lipid metabolism have provided evidence for anionic phospholipids as binding sites for aminoglycosides [1,2]. Our previous work on phospholipid-aminoglycoside interactions in vivo [3] and in vitro $[4,5]$ has specifically implicated the polyphosphoinositides, notably phosphatidylinositol bisphosphate, in such a role.

Physicochemical investigations of aminoglycoside/ lipid interactions have shown a complex pattern. Binding between the polycationic drugs and isolated phospholipids was demonstrated by a variety of techniques, but the binding affinity of the drugs did not correlate with their established toxicity [2] and neither did their ability to displace calcium from these lipids. On the other hand, increases of surface pressure of monomolecular lipid films containing phosphatidylinositol bisphosphate induced by a number of aminoglycosides demonstrated a significant correlation with ototoxicity [6]. The uniqueness of the aminoglycoside-phosphatidylinositol bisphosphate interaction may result from a specific charge orientation in the molecules leading to the formation of a complex with area-determining characteristics in monolayers [7]. It is not known, however, what impact this interaction may have on membrane characteristics such as fluidity and permeability.

Differential scanning calorimetry of liposomes is an established procedure to investigate membrane fluidity $[8,9]$. We have therefore employed this procedure to study further the interactions of the amino-

* Author to whom all correspondence should be addressed. glycoside, neomycin, with various anionic phospholipids in order to determine the effects on physicochemical properties of bilayers.

\section{MATERIALS AND METHODS}

Distearoyl L- $\alpha$-phosphatidylcholine (DSPC), L- $\alpha$ phosphatidylserine (PS), phosphatidylinositol (PI), and neomycin sulfate $(\mathrm{Nm})$, reagent grade, were purchased from the Sigma Chemical Co., St. Louis, MO. Phosphatidylinositol phosphate (PIP) and phosphatidylinositol bisphosphate $\left(\mathrm{PIP}_{2}\right)$ were isolated from Sigma brain extracts by chromatography on immobilized neomycin [10]. Lipids were stored in the dark under nitrogen at $-20^{\circ}$; PIP and PIP $_{2}$ were dried and the other lipids were in chloroformmethanol $(95: 5, \mathrm{v} / \mathrm{v})$. The purity of the lipids was greater than $95 \%$ as determined by TLC. All other chemicals were of reagent grade and the water was thrice distilled.

Preparation of liposomes. An aliquot of the chloroform-methanol $(95: 5, \mathrm{v} / \mathrm{v})$ solution of a lipid mixture was transferred to a $10-\mathrm{ml}$ round-bottom flask. The solvents were evaporated under nitrogen while the flask was rotated by hand to aid the formation of a uniformly thin lipid layer at its bottom. Residual solvent was removed by storing the flask overnight under vacuum. The dried lipid was then suspended in $0.05 \mathrm{M} \mathrm{4-(2-hydroxyethyl)-1-piperazine-ethane-}$ sulfonic acid (HFPES) ( $\mathrm{pH} 7.0, \mu=0.2$ ) by vortexing, and suspensions were centrifuged at $10,000 \mathrm{~g}$. Liposomes were resuspended in the same buffer to yield a final concentration of approximately 15 $\mu$ moles lipid $/ \mathrm{ml}$ (determined as in Ref. 11). When examined by negative strain and freeze-fracture electron microscopy, liposomes were found to be multilamellar. 
Differential scanning calorimetry (DSC). Aliquots of the vesicle suspensions were incubated at $60^{\circ}$ with various concentrations of $\mathrm{Ca}^{2+}, \mathrm{Nm}$, or both for $2 \mathrm{hr}$ to maximize penetration into the layers of the liposomes. Samples were then centrifuged, and the wet pellets were scanned in a Perkin-Elmer DSC-2C scanning calorimeter with a digitalized calorimeter output. Approximately $30 \mu \mathrm{l}$ of sample containing 0.5 to $1 \mu$ mole lipid was placed in a hermetically sealed sample pan while the reference pan contained an equal amount of buffer. All scans shown were obtained at a heating rate of $5 \% \mathrm{~min}$ and a range setting of $1 \mathrm{mcal} / \mathrm{sec}$. The calorimeter was calibrated with Indium standard (Perkin-Elmer). All experiments were repeated on at least three separate preparations.

The transition temperature $\left(T_{m}\right)$ was defined as the temperature at the apex of the transition peak, i.e. when the excess specific heat reaches a maximum. $T_{m}$ values were calculated with the Perkin-Elmer TADS, DSC Standard Software Program. This measure gave better reproducibility ( $\pm 0.2^{\circ}$ for all $T_{m}$ ), particularly with mixed lipid preparations, than the temperature of the onset of melting. Slower heating rates $\left(1^{\circ}\right.$ and $\left.2 \% \mathrm{~min}\right)$ produced identical $T_{m}$ but a slight broadening of the peak. Sharpness of the peak was also a function of the amount of lipid in the sample, but $T_{m}$ values were always within $\pm 0.2^{\circ}$ regardless of sample size.

\section{RESULTS}

DSPC liposomes. To ensure that the thermotropic changes produced by addition of $\mathrm{Ca}^{2+}$ or $\mathrm{Nm}$ to mixed liposomes were the result of interactions with the anionic component, the effects of $\mathrm{Ca}^{2+}$ and neomycin on pure DSPC liposomes were first tested. The thermogram showed the main peak at $56.7^{\circ}$ and a pretransition peak at $53.0^{\circ}$. Addition of $\mathrm{Ca}^{2+}$ or $\mathrm{Nm}$ (up to $10 \mathrm{mM}$ ) had no significant effect on the $T_{m}$ of the main transition peak. This indicated that the interactions between $\mathrm{Ca}^{2+}$ or $\mathrm{Nm}$ (at concentrations of less than $10 \mathrm{mM}$ ) and neutral phospholipids $[5,12]$ are small as compared to those with acidic lipids (see below).

PS-DSPC liposomes. $\mathrm{Ca}^{2+}$, up to $10 \mathrm{mM}$, induced a concentration-dependent increase of $T_{m}$ of PSDSPC liposomes (1:1 molar ratio) (Fig. 1A). Nm also increased $T_{m}$ but, unlike $\mathrm{Ca}^{2+}$, concentrations of $\mathrm{Nm}$ above $1 \mathrm{mM}$ caused no significant shift $\left(<0.2^{\circ}\right)$ in $T_{m}$ (Fig. 1B).

To test whether the ratio of the lipid components influenced the effect of $\mathrm{Ca}^{2+}$ and $\mathrm{Nm}$ on the mixed liposomes, mixtures of PS-DSPC in molar ratios of $1: 2$ and 2:1 were investigated (Table 1). Again. $\mathrm{Ca}^{2+}$ and $\mathrm{Nm}$ caused concentration-dependent increases in the $T_{m}$ which for the latter agent peaked at concentrations well below $10 \mathrm{mM}$. The effect of $\mathrm{Ca}^{2+}$ was more pronounced in the PS-DSPC $(2: 1)$ system. Upon addition of $10 \mathrm{mM} \mathrm{Ca}^{2+}$, an endothermic peak at the phase transition temperature of pure DSPC appeared, indicating some separation of this component from the original mixture.

Next, the effect of $\mathrm{Nm}$ in the present of $\mathrm{Ca}^{2-}$ was investigated (Table 1). When both $\mathrm{Ca}^{2+}$ and $\mathrm{Nm}$ were present at low concentration $(1 \mathrm{mM})$, the increase in $T_{m}$ approximated that induced by $\mathrm{Nm}$ alone. When $\mathrm{Ca}^{2+}$ and $\mathrm{Nm}$ were both present at $5 \mathrm{mM}$, the $T_{m}$ ranged between those induced by the individual cations. At a concentration of $\mathrm{Ca}^{2+}$ greatly exceeding that of $\mathrm{Nm}$, e.g. $10: 1$, the $T_{m}$ was closer to that found in the presence of $\mathrm{Ca}^{2+}$ alone than of $\mathrm{Nm}$ alone.

PI-DSPC liposomes. While $1 \mathrm{mM} \mathrm{Ca}^{2+}$ increased $T_{m}$ of PI-DSPC liposomes $(1: 1)$, higher concentrations of $\mathrm{Ca}^{2+}$ did not cause a further increase (Fig. 1C). $\mathrm{Nm}$ also increased $T_{m}$, and the magnitude of change was dependent on its concentration (Fig. 1D) up to $10 \mathrm{mM}$.

The addition of $\mathrm{Ca}^{2+}$ or $\mathrm{Nm}$ to PI-DSPC $(1: 2)$ and PI-DSPC (2:1) liposomes resulted in essentially similar thermotropic changes (Table 2). When $\mathrm{Ca}^{2+}$ and neomycin were both present at $1 \mathrm{mM}$, the increase in $T_{m}$ approximated or exceeded that caused by $\mathrm{Nm}$ alone. When both cations were present at $5 \mathrm{mM}$, the $T_{m}$ shifted to values between those obtained with $\mathrm{Ca}^{2+}$ and $\mathrm{Nm}$ alone in the case of PIDSPC $(1: 1)$ and PI-DSPC $(1: 2)$ mixed liposomes. However, the increase in $T_{m}$ for PI-DSPC $(2: 1)$ mixtures was close to that caused by $\mathrm{Nm}$ alone. At a ratio of $\mathrm{Ca}^{2+}: \mathrm{Nm}$ of $10: 1, \mathrm{Ca}^{2+}$ dominated, as $T_{m}$ was very close to that determined in the presence of $\mathrm{Ca}^{2+}$ alone.

PIP-DSPC liposomes. In liposomes of PIP-DSPC (1:1 molar ratio), $\mathrm{Ca}^{2+}$ caused a slight upward shift in $T_{m}$ at a $1 \mathrm{mM}$ concentration with no further effect at higher concentrations; $\mathrm{Nm}$ induced upward shifts

Table 1. Effect of $\mathrm{Ca}^{2+}$ and neomycin on phase transition of PS-DSPC liposomes

\begin{tabular}{|c|c|c|c|c|c|c|c|}
\hline \multirow[b]{3}{*}{ Concentrations } & \multicolumn{7}{|c|}{ Transition temperature $\left(T_{m}\right)\left(^{\circ}\right)$} \\
\hline & \multicolumn{2}{|c|}{$\begin{array}{c}\text { PS-DSPC }(2: 1 \text { molar ratio }) \\
\left(T_{m}=40.5^{\circ}\right)\end{array}$} & \multicolumn{3}{|c|}{$\begin{array}{c}\text { PS-DSPC }(1: 1 \text { molar ratio }) \\
\left(T_{m}=43.4^{\circ}\right)\end{array}$} & \multicolumn{2}{|c|}{$\begin{array}{c}\text { PS-DSPC }(1: 2 \text { molar ratio }) \\
\left(T_{m}=4.95^{\circ}\right)\end{array}$} \\
\hline & $\mathrm{Ca}^{2+}$ & $\mathrm{Nm}$ & $\mathrm{Ca}^{2+}$ & & $\mathrm{Nm}$ & $\mathrm{Ca}^{2+}$ & $\mathrm{Nm}$ \\
\hline $0.1 \mathrm{mM}$ & & 43.2 & & & 45.5 & & 51.2 \\
\hline $1 \mathrm{mM}$ & 41.1 & 47.3 & 44.7 & & 47.7 & 50.8 & 51.7 \\
\hline $5 \mathrm{mM}$ & 53.7 & 47.3 & 45.7 & & 47.5 & 51.1 & 53.6 \\
\hline $10 \mathrm{mM}$ & 56.0 & 47.5 & 51.2 & & 47.7 & 52.3 & 53.6 \\
\hline $1 \mathrm{mM} \mathrm{Ca}^{2+} / 1 \mathrm{mM} \mathrm{Nm}$ & \multicolumn{2}{|c|}{47.3} & \multicolumn{3}{|c|}{48.4} & \multicolumn{2}{|c|}{52.6} \\
\hline $5 \mathrm{mM} \mathrm{Ca}^{2+} / 5 \mathrm{mM} \mathrm{Nm}$ & \multicolumn{2}{|c|}{51.7} & \multicolumn{3}{|c|}{47.9} & \multicolumn{2}{|c|}{52.7} \\
\hline $10 \mathrm{mM} \mathrm{Ca}^{2+} / 1 \mathrm{mM} \mathrm{Nm}$ & \multicolumn{2}{|c|}{58.8} & \multicolumn{3}{|c|}{51.1} & \multicolumn{2}{|c|}{52.4} \\
\hline
\end{tabular}



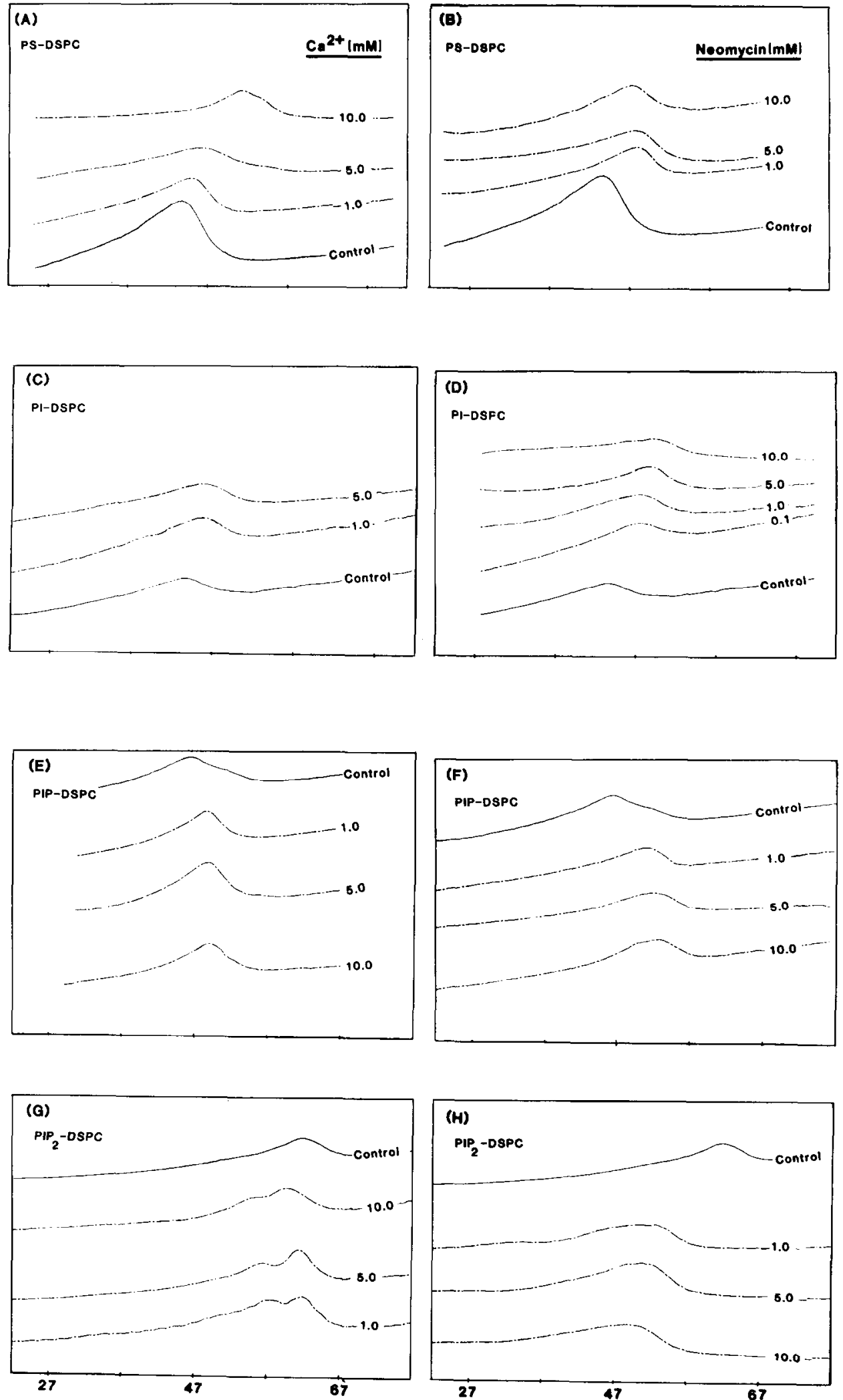

Fig. 1. Thermograms of mixed liposomes of acidic phospholipids and distearoyl L- $\alpha$-phosphatidylcholine (1:1 molar ratio) incubated with various concentrations of $\mathrm{Ca}^{2+}$ or neomycin as described in Materials and Methods. Abbreviations: DSPC, distearoyl L- $\alpha$-phosphatidylcholine; PS, phosphatidylserine; PI, phosphatidylinositol; PIP, phosphatidylinositol phosphate; PIP $_{2}$, phosphatidylinositol bisphosphate; and

$\mathrm{Nm}$, neomycin $\mathrm{B}$. 
Table 2. Effect of $\mathrm{Ca}^{2+}$ and neomycin on phase transition of PI-DSPC liposomes

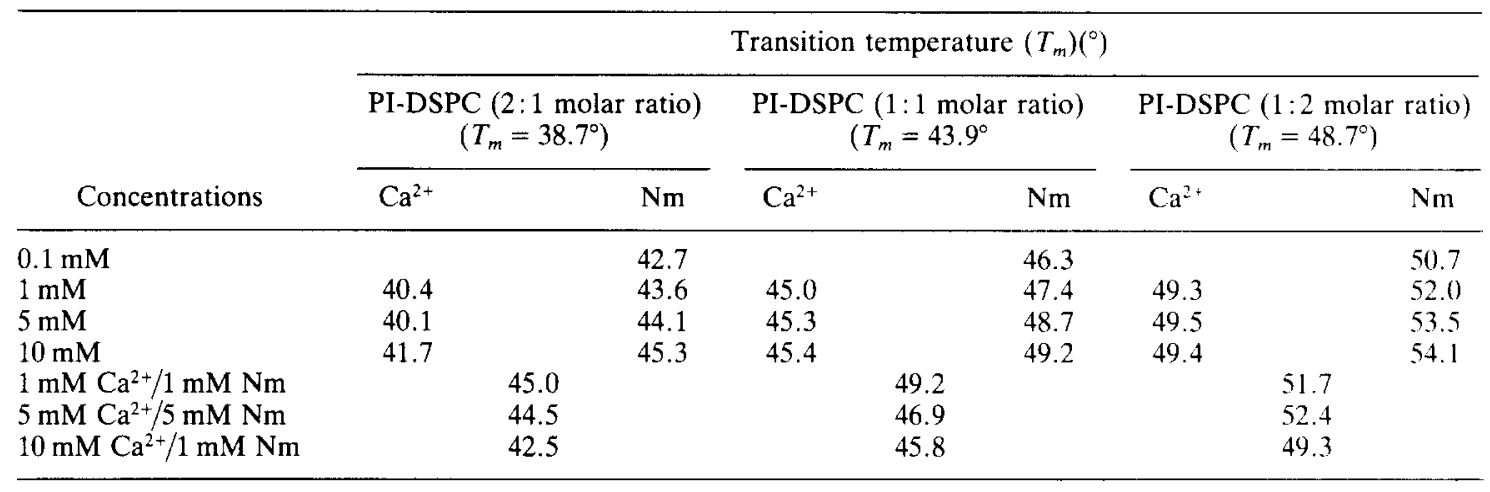

Table 3. Effect of $\mathrm{Ca}^{2+}$ and neomycin on phase transition of PIP-DSPC Liposomes*

\begin{tabular}{|c|c|c|}
\hline \multirow[b]{2}{*}{ Concentrations } & \multicolumn{2}{|c|}{$\begin{array}{l}\text { Transition temperature }\left(T_{m}\right) \\
\left(\left(^{\circ}\right)\right.\end{array}$} \\
\hline & $\mathrm{Ca}^{2+}$ & $\mathrm{Nm}$ \\
\hline $0.01 \mathrm{mM}$ & & 46.6 \\
\hline $0.1 \mathrm{mM}$ & 47.1 & 49.9 \\
\hline $1 \mathrm{mM}$ & 48.5 & 50.4 \\
\hline $5 \mathrm{mM}$ & 48.7 & 51.4 \\
\hline $10 \mathrm{mM}$ & 48.9 & 52.1 \\
\hline $1 \mathrm{mM} \mathrm{Ca}^{2+} / 1 \mathrm{mM} \mathrm{Nm}$ & \multicolumn{2}{|c|}{51.8} \\
\hline $5 \mathrm{mM} \mathrm{Ca}^{2+} / 5 \mathrm{mM} \mathrm{Nm}$ & \multicolumn{2}{|c|}{50.5} \\
\hline $10 \mathrm{mM} \mathrm{Ca}^{2+} / 1 \mathrm{mM} \mathrm{Nm}$ & \multicolumn{2}{|c|}{49.1} \\
\hline
\end{tabular}

${ }^{*}$ PIP-DSPC (1:1 molar ratio) $; T_{m}=46.1^{\circ}$.

in $T_{m}$ proportional to its concentration up to $10 \mathrm{mM}$ (Fig 1, panels $\mathrm{E}$ and $\mathrm{F}$ ).

The effect of $\mathrm{Nm}$ dominated that of $\mathrm{Ca}^{2+}$ when these ions were present in $1 \mathrm{mM}$ or $5 \mathrm{mM}$ concentrations each. The effect of $\mathrm{Ca}^{2+}$ predominated only when $\mathrm{Ca}^{2+}$ was added to a $10: 1$ excess (Table 3).

$P I P_{2}-D S P C$ liposomes. The thermogram of the PIP $_{2}$-DSPC mixed liposomes $(1: 1$ molar ratio) in the absence of $\mathrm{Ca}^{2+}$ (Fig. 1G) or $\mathrm{Nm}$ (Fig. 1H) was unlike that of the other anionic lipid-DSPC systems. The phase transition temperature was greater than that of either of its components $\left(\mathrm{PIP}_{2}=<10^{\circ}\right.$; $\mathrm{DSPC}=56.7^{\circ}$ ).

All concentrations of $\mathrm{Ca}^{2+}$ tested resulted in the appearance of a double peak (Fig. 1G). The $T_{m}$ of the first peak corresponded approximately to that of the untreated PIP $_{2}$-DSPC liposomes, and the other $T_{m}$ value approximately to that of pure DSPC. The addition of neomycin (Fig. $1 \mathrm{H}$ ) induced concentration-dependent downward shifts of the $T_{m}$. The effect of $\mathrm{Nm}$ dominated that of $\mathrm{Ca}^{2+}$ when both cations were present in equimolar ( 1 or $5 \mathrm{mM})$ concentrations (Table 4). Only in the presence of high $\mathrm{Ca}^{2+}(10 \mathrm{mM})$ and low $\mathrm{Nm}$ (1 mM) concentrations did the $T_{m}$ approximate that observed with $\mathrm{Ca}^{2+}$ alone. However, no phase separation was seen in this case.

Preparation of pure PIP and $\mathrm{PIP}_{2}$ is time consuming and costly. Since the effects of $\mathrm{Ca}^{2+}$ and $\mathrm{Nm}$ on the phase behavior of other mixed liposomes were essentially independent of the molar ratio, we initially limited experiments with PIP-DSPC and $\mathrm{PIP}_{2}$-DSPC liposomes to 1:1 molar ratios. However, a few experiments were run with PIP $_{2}$-DSPC $(2: 1$ molar ratio) liposomes. In both cases, the results were consistent: $T_{m}$ was higher than those of the individual components and $\mathrm{Ca}^{2+}$ or $\mathrm{Nm}$ reduced $T_{m}$ (Table 4).

Table 4. Effect of $\mathrm{Ca}^{2+}$ and neomycin on phase transition of $\mathrm{PIP}_{2}$-DSPC liposomes

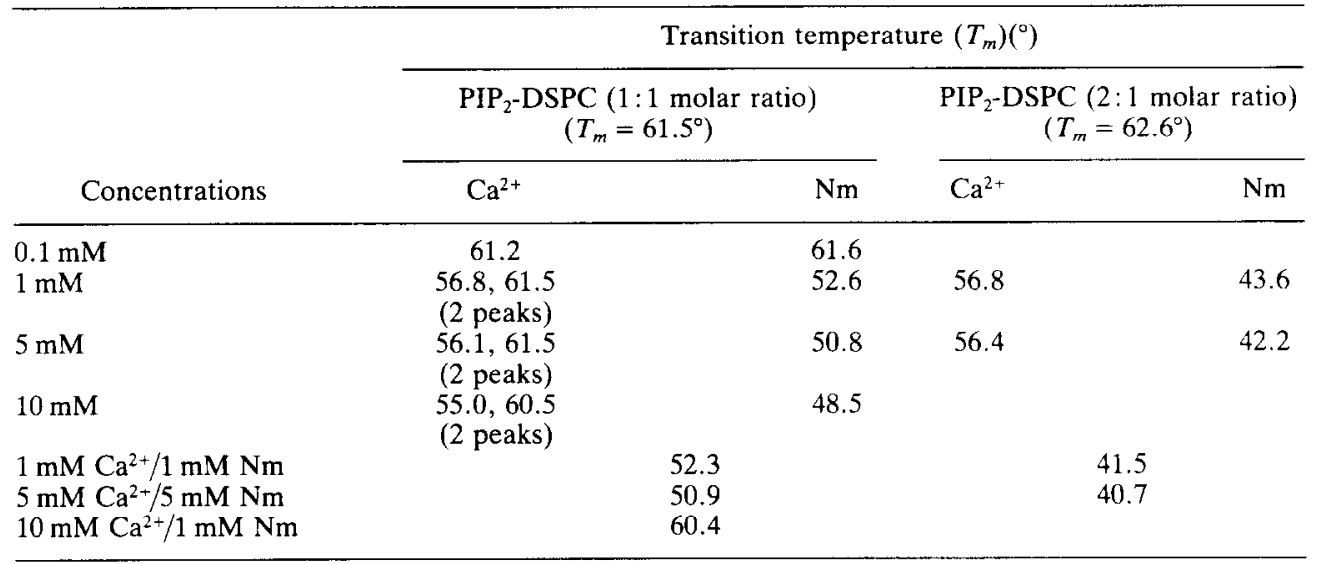




\section{DISCUSSION}

The thermotropic behavior of the PIP $_{2}$-DSPC liposomes differed from that of the other lipids tested. First, their $T_{m}$ was greater than that of either of its components, a behavior which was not seen with any of the other mixtures. While unusual, a few lipid mixtures have been demonstrated to have a $T_{m}$ higher than that of either component, possibly due to the formation of hydrogen bonds between available hydroxyl and the phosphate groups [13-15]. Similar hydrogen bonding may exist in $\mathrm{PIP}_{2}$ as this lipid is not fully ionized at neutral $\mathrm{pH}$ due to the high surface charge density [16]. There is also some evidence that hydrogen bonding may occur bctween the hydroxyl groups in the inositol molecules [14]. Intermolecular hydrogen bonding between the phosphate group in DSPC and the phosphate groups and/or the hydroxyl groups in $\mathrm{PIP}_{2}$ molecules is then possible. The existence of such bonds should raise the transition temperature since the gel state is more stabilized by these forces than is the liquid-crystalline state.

Second, the induction of the double peak by $\mathrm{Ca}^{2+}$ was seen in these liposomes only. This is consistent with previous findings that $\mathrm{Ca}^{2+}$ has a specially strong affinity to $\mathrm{PIP}_{2}[16]$, and suggests that molecular segregation is occurring within the vesicles [17]. Other interpretations, however, cannot be ruled out. The downward shift of $T_{m}$ induced by $\mathrm{Nm}$ is compatible with the results obtained from monolayer studies, which suggested the formation of a strong ionic complex between $\mathrm{Nm}$ and $\mathrm{PIP}_{2}$ with a concomitant increase in surface pressure [5]. Similarly, proteins which bind primarily by ionic bonds to phospholipids and increase surface pressure of monolayers produce a substantial decrease in $T_{m}$ [9].

In contrast to PIP $_{2}$, the behavior of other anionic lipids follows conventional patterns. Both $\mathrm{Ca}^{2+}$ and $\mathrm{Nm}$ increased $T_{m}$ of mixed PS-DSPC liposomes. $\mathrm{Nm}$ appeared to be more effective at low concentrations $(1 \mathrm{mM})$ whereas $\mathrm{Ca}^{2+}$ was far more effective at higher concentrations, particularly in PS-rich liposomes. Based on electrical considerations alone, it is not surprising that $\mathrm{Nm}(+6)$ affects the $T_{m}$ of the negatively charged liposomes more than $\mathrm{Ca}^{2+}$. The prevalence of $\mathrm{Ca}^{2+}$ at higher concentrations can be the result of a specific binding of $\mathrm{Ca}^{2+}$ to PS $[18,19]$. In contrast, $\mathrm{Nm}$ was more effective than $\mathrm{Ca}^{2+}$ in both PI-DSPC and PIP-DSPC liposomes. This is consistent with results by Ohki et al. [20], who reported little or no phase separation for $\mathrm{Ca}^{2+}$. treated PI-PC systems. Although $\mathrm{Nm}$ was more effective than $\mathrm{Ca}^{2+}$ at all concentrations tested, it should be pointed out that separation into a phase very rich in DSPC (appearance of a peak corresponding to pure DSPC) was not observed for either PI-DSPC or PIP-DSPC systems even at Nm concentrations of $10 \mathrm{mM}$.

The behavior of PIP $_{2}$-enriched liposomes may be the result of a unique interaction of $\mathrm{Ca}^{2+}$ or $\mathrm{Nm}$ with this lipid. The present study alone does not allow such a conclusion, as other interpretations are possible. For instance, since liposomes are multi- lamellar, it is unknown what fraction of the bilayer interacts with cations and whether this fraction is affected by the composition of the liposomes. However, if we consider our previous in vivo [4] and in vitro $[5,6]$ studies, the present results do confirm the hypothesis that $\mathrm{PIP}_{2}$ occupies a unique role among acidic phospholipids in its molecular interactions with $\mathrm{Ca}^{2+}$ and with the aminoglycosides. What are the implications of such an interaction for a biological system? The $\mathrm{Ca}^{2+}$-induced phase separation in $\mathrm{PIP}_{2}-$ enriched liposomes may be indicative of an important control mechanism in membranes. For instance, it can reduce the lateral diffusion in the aggregates and the transverse movement of molecules and ions across the membranc. Nm acted antagonistically and lowered $T_{m}$. This fluidizing effect of $\mathrm{Nm}$ and the antagonism to calcium may critically alter permeability and other properties of biological membranes. These results then support and extend our previous findings and conclusions that $\mathrm{PIP}_{2}$ plays a crucial role in the expression of aminoglycoside toxicity

Acknowledgement-This research was supported by Grant NS-13792 and Program Project Grant NS-05785 from the National Institutes of Health.

\section{REFERENCES}

1. H. D. Humes, N. D. Weiner and J. Schacht, in Nephrotoxicity and Ototoxicity of Drugs (Ed. J. P. Fillastre), p. 333. Editions INSERM, Paris (1982).

2. M. Sastrasinh, T. C. Knauss, J. M. Weinberg and H. D. Humes, J. Pharmac. exp. Ther. 222, 350 (1982).

3. A. Orsulakova, E. Stockhorst and J. Schacht, J. Neurochem. 26, 285 (1976).

4. J. Schacht, Archs Oto-Rhino-Laryngol. 224, 129 (1979)

5. S. Lodhi, N. D. Weiner and J. Schacht, Biochim. biophys. Acta 557, 1 (1979).

6. S. Lodhi, N. D. Weiner, I. Mechigian and J. Schacht Biochem. Pharmac. 29, 597 (1980).

7. J. Schacht, S. Lodhi and N. D. Weiner, in Membrane Toxicity (Eds. M. W. Miller and A. E. Shamoo), p. 191. Plenum Press, New York (1977).

8. D. Papahadjopoulos, K. Jacobson, G. Poste and G. Shepherd, Biochim. biophys. Acta 394, 504 (1975).

9. D. Papahadjopoulos, J. Colloid Interface Sci. 58, 459 (1977).

10. J. Schacht, J. Lipid Res. 19, 1063 (1978).

11. C. H. Fiske and Y. Subba Row, J. biol. Chem. 66, 375 (1925).

12. M. G. Ganesan, D. L. Schwinke and N. Werner, Biochim. biophys. Acta 686, 245 (1982)

13. H. Eibl and P. Wooley, Biophys. Chem. 10, 261 (1979).

14. J. M. Boggs, Can. J. Biochem. 58, 755 (1980).

15. S. Massari, D. Pascolini and G. Gradenigo, Biochemistry 17, 4465 (1978).

16. H. Hauser and R. M. C. Dawson, Eur. J. Biochem. 1, 61 (1967).

17. D. Papahadjopoulos, G. Poste, B. E. Shaeffer and W. T. Vail, Biochim. biophys. Acta 252, 10 (1974).

18. K. Jacobson and D. Papahadjopoulus, Biochemistry 14, 152 (1975).

19. S. Ohnishi and T. Ito, Biochemistry 13, 881 (1974).

20. K. Ohki, S. Takashi, T. Yanauchi and Y. Nozawa, Biochim. biophys. Acta 644, 165 (1981). 